\title{
Flavour structure and proton decay in 6D orbifold GUTs
}

\author{
Wilfried Buchmüller \\ Deutsches Elektronen-Synchrotron DESY \\ Hamburg, Germany \\ E-mail: buchmuwi@mail.desy.de

\section{Laura Covi} \\ Theory Division, CERN Department of Physics \\ Geneva, Switzerland \\ E-mail: laura.covi@cern.ch

\section{David Emmanuel-Costa} \\ CFTP, Departamento de Fúsica, Instituto Superior Técnico \\ Lisbon, Portugal \\ E-mail: emmanuel@mail.desy.de
}

\section{Sören Wiesenfeldt}

Deutsches Elektronen-Synchrotron DESY

Hamburg, Germany

E-mail: soeren@mail.desy.de

ABSTRACT: We study proton decay in a supersymmetric $\mathrm{SO}(10)$ gauge theory in six dimensions compactified on an orbifold. The dimension- 5 proton decay operators are forbidden by R-symmetry, whereas the dimension- 6 operators are enhanced due to the presence of KK towers. Three sequential quark-lepton families are localised at the three orbifold fixed points, where $\mathrm{SO}(10)$ is broken to its three GUT subgroups. The physical quarks and leptons are mixtures of these brane states and additional bulk zero modes. This leads to a characteristic pattern of branching ratios in proton decay, in particular the suppression of the $p \rightarrow \mu^{+} K^{0}$ mode.

Keywords: Field Theories in Higher Dimensions, GUT, 


\section{Contents}

1. Introduction 1

2. 6D orbifold GUT model 2

3. Proton decay via dimension-6 operators 6

3.1 Effective $\mathrm{SU}(5)$ operators in $4 \mathrm{D}$ models

3.2 Effective operators in $6 \mathrm{D}$ models

3.3 Corrections from derivative brane operators

4. Flavour structure and branching ratios

4.1 Flavour mixing in 6D versus 4D GUT models 10

4.2 Decay rates and branching ratios

5. Conclusions 15

\section{Introduction}

The most striking consequence of Grand Unified Theories is proton decay [ [ $]$ - [6]. It has been predicted and sought for more than 30 years, and its absence constitutes a very strong constraint on any realistic GUT model.

Recently, the discussion of proton decay has been revitalised on two different fronts. On the experimental side, the bounds coming from the SuperKamiokande experiment have reached $\tau\left(p \rightarrow e^{+} \pi^{0}\right) \geq 5.3 \times 10^{33}$ yrs [7] and $\tau\left(p \rightarrow K^{+} \bar{\nu}\right) \geq 1.9 \times 10^{33}$ yrs [8]. On the theoretical side, these bounds have motivated new detailed studies of proton decay via dimension-5 operators in supersymmetric GUTs [9]-[14]. These analyses showed that the present bounds disfavour this class of models, although the theoretical predictions strongly depend on the flavour structure assumed [12]-[14].

Dimension-6 operators are less dangerous and have not drawn so much attention in the literature. It is, however, well known that such operators depend on the flavour structure as well [15]- 17], and it has been realised that the observed leptonic mixing can have a strong effect on the proton decay branching ratios [18]. Recently, dimension-6 operators have been studied in flipped $\mathrm{SU}(5)$ [19] and $\mathrm{SO}(10)$ [18, 20] GUTs.

The interest in dimension-6 operators has been renewed by GUT models in higher dimensions, with symmetry breaking via orbifolds. Here dimension-5 proton decay is naturally absent [21, 22. In such models though, the dimension- 6 operators are enhanced compared to the usual 4D case, due to the lower mass scale for the heavy particles mediating the decay and the presence of Kaluza-Klein towers of such states [22]-[24]. Furthermore, the proton decay branching ratios depend on the localisation of quarks and leptons [23, 24]. 
The goal of the present paper is to study proton decay via dimension- 6 operators in a specific $\mathrm{SO}(10)$ orbifold GUT model in $6 \mathrm{D}$, where the complete Standard Model flavour structure can be reproduced via mixing of brane states with bulk split multiplets [25]. This kind of model can naturally arise in compactifications of the heterotic string [26 27]. Quark and lepton mass matrices are approximately of lopsided type, with some characteristic modifications compared to the 4D lopsided picture. The up quark of the first generation is located on a brane where the bulk gauge group $\mathrm{SO}(10)$ is broken to $\mathrm{SU}(5) \times \mathrm{U}(1)$, and therefore $\mathrm{SU}(5)$ gauge boson exchange gives the main contribution to proton decay. For this model, we will calculate the total rate and the branching ratios of proton decay and compare the results with those in $4 \mathrm{D} \mathrm{SU}(5)$ models with $\mathrm{U}(1)$ family symmetry.

The paper is organised as follows: in section 2, we discuss the flavour structure of the $6 \mathrm{D}$ orbifold GUT model and evaluate the mixing matrices needed to calculate proton decay widths. Section 3 deals with proton decay via dimension- 6 operators in the usual 4D case as well as the 6D model for which the sum over the Kaluza-Klein tower is performed. Finally, in section 4 we discuss the effects of the flavour structure on the proton decay branching ratios in the different models. Conclusions are given in section 0 .

\section{6D orbifold GUT model}

We start from an $\mathrm{SO}(10)$ gauge theory in $6 \mathrm{D}$ with $N=1$ supersymmetry compactified on the orbifold $T^{2} /\left(Z_{2}^{I} \times Z_{2}^{\mathrm{PS}} \times Z_{2}^{\mathrm{GG}}\right)[28,29]$. The theory has four fixed points, $O_{\mathrm{I}}, O_{\mathrm{PS}}, O_{\mathrm{GG}}$ and $O_{\mathrm{fl}}$, located at the four corners of a 'pillow' corresponding to the two compact dimensions (cf. figure 1). At $O_{\mathrm{I}}$, only SUSY is broken, whereas at the other fixed points, $O_{\mathrm{PS}}, O_{\mathrm{GG}}$ and $O_{\mathrm{fl}}$, also $\mathrm{SO}(10)$ is broken to its three GUT subgroups $\mathrm{G}_{\mathrm{PS}}=\mathrm{SU}(4) \times \mathrm{SU}(2) \times \mathrm{SU}(2)$ [1], $\mathrm{G}_{\mathrm{GG}}=\mathrm{SU}(5) \times \mathrm{U}(1)_{X}$ [2] and flipped $\mathrm{SU}(5)$ [5, [], $\mathrm{G}_{\mathrm{fl}}=\mathrm{SU}(5)^{\prime} \times \mathrm{U}(1)^{\prime}$, respectively. The intersection of all these GUT groups yields the standard model group with an additional $\mathrm{U}(1)$ factor, $\mathrm{G}_{\mathrm{SM}^{\prime}}=\mathrm{SU}(3) \times \mathrm{SU}(2) \times \mathrm{U}(1)_{Y} \times \mathrm{U}(1)_{Y^{\prime}}$, as unbroken gauge symmetry below the compactification scale.

The field content of the theory is strongly constrained by imposing the cancellation of irreducible bulk and brane anomalies [30, 31. We study the model proposed in [25], containing three 16-plets $\psi_{i}, i=1, \ldots, 3$, as brane fields, and six 10-plets, $H_{1}, \ldots, H_{6}$, and four 16-plets, $\Phi, \Phi^{c}, \phi, \phi^{c}$, as bulk hypermultiplets. Vacuum expectation values of $\Phi$ and $\Phi^{c}$ break the surviving $\mathrm{U}(1)_{B-L}$. The electroweak gauge group is broken by expectation values of the anti-doublet and doublet contained in $H_{1}$ and $H_{2}$.

We choose the parities of $H_{5}, H_{6}$ and $\phi, \phi^{c}$ such that their zero modes are

$$
L=\left(\begin{array}{c}
\nu_{4} \\
e_{4}
\end{array}\right), \quad L^{c}=\left(\begin{array}{c}
\nu_{4}^{c} \\
e_{4}^{c}
\end{array}\right), \quad G_{5}^{c}=d_{4}^{c}, \quad G_{6}=d_{4} .
$$

These zero modes act as a (vectorial) fourth generation of down quarks and leptons and mix with the three generations of brane fields. We allocate the three sequential 16-plets to the three branes where $\mathrm{SO}(10)$ is broken to its three GUT subgroups, and place $\psi_{1}$ at $O_{\mathrm{GG}}, \psi_{2}$ at $O_{\mathrm{fl}}$ and $\psi_{3}$ at $O_{\mathrm{Ps}}$. The three 'families' are then separated by distances large compared to the cutoff scale $M_{*}$. Hence, they can only have diagonal Yukawa couplings 


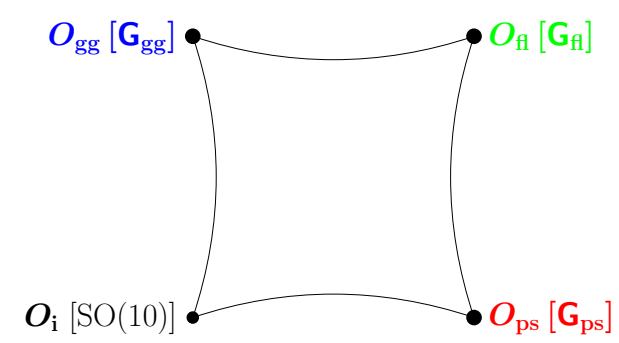

Figure 1: The three $\mathrm{SO}(10)$ subgroups at the corresponding fixed points (branes) of the orbifold $T^{2} / Z_{2} \times Z_{2}^{\prime} \times Z_{2}^{\prime \prime}$.

with the bulk Higgs fields. Direct mixings are exponentially suppressed. The brane fields, however, can mix with the bulk zero modes for which we expect no suppression. These mixings take place only among left-handed leptons and right-handed down-quarks. This leads to a characteristic pattern of mass matrices.

As described in [25], after $B-L$ breaking at the scale $v_{N}$ and electroweak symmetry breaking via $v_{1}=\left\langle H_{1}^{c}\right\rangle, v_{2}=\left\langle H_{2}\right\rangle$, the mass terms assume the characteristic form

$$
W=d_{\alpha} m_{\alpha \beta}^{d} d_{\beta}^{c}+e_{\alpha}^{c} m_{\alpha \beta}^{e} e_{\beta}+n_{\alpha}^{c} m_{\alpha \beta}^{D} \nu_{\beta}+u_{i} m_{i}^{u} u_{i}^{c}+\frac{1}{2} n_{i}^{c} m_{i}^{N} n_{i}^{c},
$$

where the Greek indices, $\alpha=1 \ldots 4$, include also the bulk states with $n_{4}^{c}=\nu_{4}^{c}$, while Latin indices only run over brane fields, $i=1 \ldots 3$. Note that there is no Majorana mass for $n_{4}^{c}=\nu_{4}^{c}$ which originates from the $6 \mathrm{D}$ hypermultiplet. $m^{u}$ and $m^{N}$ are diagonal $3 \times 3$ matrices $\left(\tan \beta=v_{2} / v_{1}\right)$,

$$
\frac{1}{\tan \beta} m^{u} \sim \frac{v_{1} M_{*}}{v_{N}^{2}} m^{N} \sim\left(\begin{array}{ccc}
\mu_{1} & 0 & 0 \\
0 & \mu_{2} & 0 \\
0 & 0 & \mu_{3}
\end{array}\right),
$$

whereas $m^{d}, m^{e}$ and $m^{D}$ are $4 \times 4$ matrices with the common structure,

$$
\frac{1}{\tan \beta} m^{D} \sim m^{d} \sim m^{e} \sim\left(\begin{array}{cccc}
\mu_{1} & 0 & 0 & \widetilde{\mu}_{1} \\
0 & \mu_{2} & 0 & \widetilde{\mu}_{2} \\
0 & 0 & \mu_{3} & \widetilde{\mu}_{3} \\
\widetilde{M_{1}} & \widetilde{M}_{2} & \widetilde{M}_{3} & \widetilde{M}_{4}
\end{array}\right) \equiv m .
$$

Here $\mu_{i}, \widetilde{\mu}_{i}=\mathcal{O}\left(v_{1}\right)$ and $\widetilde{M}_{i}=\mathcal{O}\left(\Lambda_{G U T}\right)$. The diagonal and the off-diagonal elements of these matrices satisfy several relations due to the underlying GUT symmetries [25]. The hypothesis of a universal strength of Yukawa couplings at each fixpoint leads to the identification of the diagonal and off-diagonal elements of $m^{u} / \tan \beta, m^{d}, m^{e}$ and $m^{D} / \tan \beta$ up to coefficients of order one. This implies an approximate top-bottom unification with large $\tan \beta$ and a parametrisation of quark and lepton mass hierarchies in terms of the six parameters $\mu_{1}, \mu_{2}, \mu_{3}$ and $\widetilde{\mu}_{1}, \widetilde{\mu}_{2}, \widetilde{\mu}_{3}$.

The crucial feature of the matrices $m^{d}, m^{e}$ and $m^{D}$ are the mixings between the six brane states and the two bulk states. The first three rows of the matrices are proportional to the electroweak scale. The corresponding Yukawa couplings have to be hierarchical in 
order to obtain a realistic spectrum of quark and lepton masses. In a complete theory, this hierarchy may be due to the different location of the fixpoints on the orbifold. The fourth row, $\widetilde{M}_{\alpha}$, is of order the unification scale and, as we assume, non-hierarchical.

The mass matrix $m$ (cf. eq. (2.4) ) can be diagonalised by the unitary transformation

$$
m=U_{4} U_{3} D V_{3}^{\dagger} V_{4}^{\dagger},
$$

where the matrices $U_{4}, V_{4}$ single out the heavy mass eigenstate, that can then be integrated out, while the matrices $U_{3}, V_{3}$ act only on the SM flavour indices and perform the final diagonalisation in the remaining $3 \times 3$ subspace. $U_{4}$ and $V_{4}$ are given by (neglecting phases)

$$
\begin{aligned}
U_{4}= & \left(\begin{array}{cccc}
1 & 0 & 0 & \frac{\mu_{1} \widetilde{M}_{1}+\widetilde{\mu}_{1} \widetilde{M}_{4}}{\widetilde{M}^{2}} \\
0 & 1 & 0 & \frac{\mu_{2} \widetilde{M}_{2}+\widetilde{\mu}_{2} \widetilde{M}_{4}}{\widetilde{M}^{2}} \\
0 & 0 & 1 & \frac{\mu_{3} \widetilde{M}_{3}+\widetilde{\mu}_{3} \widetilde{M}_{4}}{\widetilde{M}^{2}} \\
-\frac{\mu_{1} \widetilde{M}_{1}+\widetilde{\mu}_{1} \widetilde{M}_{4}}{\widetilde{M}^{2}} & -\frac{\mu_{2} \widetilde{M}_{2}+\widetilde{\mu}_{2} \widetilde{M}_{4}}{\widetilde{M}^{2}}-\frac{\mu_{3} \widetilde{M}_{3}+\widetilde{\mu}_{3} \widetilde{M}_{4}}{\widetilde{M}^{2}} & 1
\end{array}\right)+\mathcal{O}\left(\frac{v^{2}}{\widetilde{M}^{2}}\right) \\
V_{4}= & \left(\begin{array}{cccc}
\frac{\widetilde{M}_{4}}{\widetilde{M}_{14}} & 0 & -\frac{\widetilde{M}_{1} \widetilde{M}_{23}}{\widetilde{M} \widetilde{M}_{14}} & \frac{\widetilde{M}_{1}}{M} \\
0 & \frac{\widetilde{M}_{3}}{\widetilde{M}_{23}} & \frac{\widetilde{M}_{2} \widetilde{M}_{14}}{\widetilde{M} \widetilde{M}_{23}} & \frac{\widetilde{M}_{2}}{M} \\
0 & -\frac{\widetilde{M}_{2}}{\widetilde{M}_{23}} & \frac{\widetilde{M}_{3} \widetilde{M}_{14}}{\widetilde{M} \widetilde{M}_{23}} & \frac{\widetilde{M}_{3}}{\widetilde{M}} \\
-\frac{\widetilde{M}_{1}}{\widetilde{M}_{14}} & 0 & -\frac{\widetilde{M}_{4} \widetilde{M}_{23}}{\widetilde{M} \widetilde{M}_{14}} & \frac{\widetilde{M}_{4}}{M}
\end{array}\right)
\end{aligned}
$$

where $\widetilde{M}=\sqrt{\sum_{\alpha} \widetilde{M}_{\alpha}^{2}}$ and $\widetilde{M}_{\alpha \beta}=\sqrt{\widetilde{M}_{\alpha}^{2}+\widetilde{M}_{\beta}^{2}}$. In general, $V_{4}$ contains large mixings, while $U_{4}$ is approximately the unit matrix up to corrections $\mathcal{O}(v / \widetilde{M}) . U_{3}$ and $V_{3}$ are the matrices that diagonalise

$$
m^{\prime}=U_{4}^{\dagger} m V_{4}=\left(\begin{array}{cc}
\widehat{m} & 0 \\
0 & \widetilde{M}
\end{array}\right)+\mathcal{O}\left(\frac{v^{2}}{\widetilde{M}}\right),
$$

where

$$
\widehat{m}=\left(\begin{array}{l}
\mu_{1}\left(V_{4}\right)_{1 j}+\widetilde{\mu}_{1}\left(V_{4}\right)_{4 j} \\
\mu_{2}\left(V_{4}\right)_{2 j}+\widetilde{\mu}_{2}\left(V_{4}\right)_{4 j} \\
\mu_{3}\left(V_{4}\right)_{3 j}+\widetilde{\mu}_{3}\left(V_{4}\right)_{4 j}
\end{array}\right) .
$$

Clearly, they have only a non-trivial $3 \times 3$ part,

$$
U_{3}=\left(\begin{array}{cc}
V_{\text {СКм }}^{\dagger} & 0 \\
0 & 1
\end{array}\right), \quad V_{3}=\left(\begin{array}{ll}
\widehat{V} & 0 \\
0 & 1
\end{array}\right) .
$$

Notice that the rows of $\widehat{m}$ scale each like $\mu_{i}, \widetilde{\mu}_{i}$. Hence, for hierarchical parameters $\mu_{1}, \widetilde{\mu}_{1}<$ $\mu_{2}, \widetilde{\mu}_{2}<\mu_{3}, \widetilde{\mu}_{3}$ we obtain a structure familiar from lopsided fermion mass models.

As discussed in [25], we can choose the parameters in such a way to give a consistent quark mass pattern and CKM matrix, in particular

$$
\mu_{1}: \mu_{2}: \mu_{3} \sim m_{u}: m_{c}: m_{t},
$$


and (cf. eq. (2.17))

$$
\bar{\mu}_{3} \simeq m_{b}, \quad \widetilde{\mu}_{2}: \bar{\mu}_{3} \sim m_{s}: m_{b} .
$$

The CKM matrix in $U_{3}$ arises in a natural way as well. Setting the remaining parameter $\widetilde{\mu}_{1}$ to give

$$
V_{u s}=\Theta_{c} \simeq \frac{\widetilde{\mu}_{1}}{\widetilde{\mu}_{2}} \simeq 0.2,
$$

the other matrix elements are determined by the quark masses [25,

$$
V_{c b} \sim \frac{\widetilde{\mu}_{2}}{\widetilde{\mu}_{3}} \simeq \frac{m_{s}}{m_{b}} \simeq 2 \times 10^{-2}, \quad V_{u b} \sim \frac{\widetilde{\mu}_{1}}{\widetilde{\mu}_{3}}=\Theta_{c} \frac{m_{s}}{m_{b}} \simeq 4 \times 10^{-3} .
$$

Within the accuracy of our approach this is consistent with the analysis from weak decays [32].

The matrix $\widehat{V}$ takes a relatively simple form in the case when $\mu_{1,2}=0$ and the mass matrix in eq. (2.9) has one zero eigenvalue. We then obtain

$$
\widehat{V}=\left(\begin{array}{ccc}
-\frac{\widetilde{M}_{2} \widetilde{M}_{4}}{\widetilde{M}_{12} \widetilde{M}_{14}} & \frac{\widetilde{M}_{1}\left(\widetilde{\mu}_{3} \widetilde{M}_{3} \widetilde{M}_{4}-\mu_{3}\left(\widetilde{M}_{1}^{2}+\widetilde{M}_{2}^{2}+\widetilde{M}_{4}^{2}\right)\right)}{\bar{\mu}_{3} \widetilde{M} \widetilde{M}_{12} \widetilde{M}_{14}} & -\frac{\widetilde{\mu}_{3}}{\bar{\mu}_{3}} \frac{\widetilde{M}_{1}}{\widetilde{M}_{14}} \\
\frac{\widetilde{M}_{1} \widetilde{M}_{3}}{\widetilde{M}_{12} \widetilde{M}_{23}} & \frac{\widetilde{M}_{2}\left(\widetilde{\mu}_{3}\left(\widetilde{M}_{1}^{2}+\widetilde{M}_{2}^{2}+\widetilde{M}_{3}^{2}\right)-\mu_{3} \widetilde{M}_{3} \widetilde{M}_{4}\right)}{\bar{\mu}_{3} \widetilde{M} \widetilde{M}_{12} \widetilde{M}_{23}} & -\frac{\mu_{3}}{\bar{\mu}_{3}} \frac{\widetilde{M}_{2}}{\widetilde{M}_{23}} \\
\frac{\widetilde{M}_{1} \widetilde{M}_{2} \widetilde{M}}{\widetilde{M}_{12} \widetilde{M}_{14} \widetilde{M}_{23}} & -\frac{\widetilde{\mu}_{3} \widetilde{M}_{1}^{2} \widetilde{M}_{3}+\mu_{3} \widetilde{M}_{2}^{2} \widetilde{M}_{4}}{\bar{\mu}_{3} \widetilde{M}_{12} \widetilde{M}_{14} \widetilde{M}_{23}} & -\frac{\widetilde{\mu}_{3}}{\bar{\mu}_{3}} \frac{\widetilde{M}_{4} \widetilde{M}_{23}}{\bar{M} \widetilde{M}_{14}}+\frac{\mu_{3}}{\bar{\mu}_{3}} \frac{\widetilde{M}_{3} \widetilde{M}_{14}}{M M_{23}}
\end{array}\right),
$$

up to a two-dimensional mixing matrix for the second and third generation, parameterized by a small angle $\Theta_{R}$,

$$
\Theta_{R} \simeq \frac{\widetilde{\mu}_{1}^{2}+\widetilde{\mu}_{2}^{2}}{\bar{\mu}_{3}^{2}} \ll 1
$$

where we have defined

$$
\bar{\mu}_{3}^{2}=\widetilde{\mu}_{3}^{2}\left(1-\frac{\widetilde{M}_{4}^{2}}{\widetilde{M}^{2}}\right)+\mu_{3}^{2}\left(1-\frac{\widetilde{M}_{3}^{2}}{\widetilde{M}^{2}}\right)-2 \mu_{3} \widetilde{\mu}_{3} \frac{\widetilde{M}_{3} \widetilde{M}_{4}}{\widetilde{M}^{2}} .
$$

The case of small $\mu_{1,2}$ limit is actually of physical relevance, since it gives for the down quark

$$
\frac{m_{d}}{m_{s}} \sim \frac{\mu_{2}}{\widetilde{\mu}_{2}} \frac{\widetilde{\mu}_{1}}{\widetilde{\mu}_{2}} \sim \Theta_{c} \frac{m_{c} m_{b}}{m_{t} m_{s}} \simeq 0.03
$$

consistent with data [33]. Since $\mu_{2} / \widetilde{\mu}_{2} \sim m_{c} m_{b} /\left(m_{t} m_{s}\right) \sim 0.1$, the corrections to the matrix (2.15) are small.

The charged lepton mass matrix $m^{e}$ has the same structure as the down-quark mass matrix, but there the large mixings are between the 'left-handed' states $e_{i}$. Experimental data require for the largest eigenvalue $m_{\tau} \simeq m_{b}$, whereas the second and the third eigenvalue have to satisfy the relations $m_{\mu} \simeq 3 m_{s}$ and $m_{e} \simeq 0.2 m_{d}$, respectively. This is consistent with our identification of $m^{d}$ and $m^{e}$ up to coefficients of order one unless the relevant parameters are fixed by GUT relations. The comparision between the expression (2.18) for $m_{d}$ and the corresponding one for $m_{e}$ suggests locating the second 
family on the flipped $\mathrm{SU}(5)$ brane. The successful relation for the light neutrino mass, $m_{3} \sim m_{t}^{2} / M_{3} \sim m_{t}^{2} M_{*} / v_{N}^{2} \sim 0.01 \mathrm{eV}$, requires the third family to be located on the PS brane. With the first family on the GG brane, $m_{d}$ and $m_{e}$ are determined by the parameters $\mu_{2}^{d}$ and $\mu_{2}^{e}$, which are not related by a flipped SU(5) mass relation.

\section{Proton decay via dimension-6 operators}

\subsection{Effective SU(5) operators in $4 \mathrm{D}$ models}

Dimension-6 proton decay in the SU(5) model is mediated by the exchange of the $X$ and $Y$ leptoquark gauge bosons [17. Contrary to the dimension-5 operator, it does not involve any dressing through supersymmetric partners and therefore it is not sensitive to the supersymmetry breaking scale (except for the weak dependence of the GUT scale on the superparticle mass spectrum). The effective vertex is obtained by simply integrating out the heavy gauge bosons.

The couplings of the $\mathrm{SU}(5)$ representations $\mathbf{5}^{*}$ and $\mathbf{1 0}$ with the $\mathrm{SU}(5)$ gauge bosons are given by their kinetic terms,

$$
\int_{\theta^{2} \bar{\theta}^{2}} \sum_{\text {reps }} \bar{\Phi}_{i} e^{2 V} \Phi_{i}
$$

which include

$$
\mathcal{L}=i \frac{g_{5}}{\sqrt{2}} A_{\mu}^{a}\left[2 \operatorname{tr}\left(\overline{\mathbf{1 0}}_{\mathbf{i}} \gamma^{\mu} T^{a} \mathbf{1 0}_{\mathbf{i}}\right)+\overline{\mathbf{5}}_{\mathbf{k}}^{*} \gamma^{\mu}\left(T^{a}\right)^{\top} \mathbf{5}_{\mathbf{k}}^{*}\right]+\text { h.c. },
$$

where $g_{5}$ is the $\mathrm{SU}(5)$ gauge coupling; the Latin indices count the generations, $i=1 \ldots 3$ those of $\mathbf{1 0}, k=1 \ldots 3$ those of $\mathbf{5}^{*}$.

We now express the $\mathrm{SU}(5)$ representations in terms of SM fields with $\mathcal{X}=(X, Y)$ being a $\left(\mathbf{3}^{*}, \mathbf{2}, 5 / 6\right)$ representation of $\mathrm{SU}(3) \times \mathrm{SU}(2) \times \mathrm{U}(1)$. This yields the baryon and lepton number violating terms

$$
\mathcal{L}=-i \frac{g_{5}}{\sqrt{2}} \mathcal{X}_{\alpha \mu}\left[\epsilon_{\alpha \beta \gamma} \bar{Q}_{\beta, i} \gamma^{\mu} u_{\gamma, i}^{c}+{\overline{e^{c}}}_{i} \gamma^{\mu} Q_{\alpha, i}-\overline{d^{c}}{ }_{\alpha, k} \gamma^{\mu} L_{k}\right]+\text { h.c. },
$$

where Greek indices denote the colour degrees of freedom and the $\mathrm{SU}(2)$ indices have been suppressed. Note that the first two terms come from the $\mathbf{1 0}$ representation, the last one from the $\mathbf{5}^{*}$.

Integrating out the heavy gauge bosons with masses $M_{\mathcal{X}}$, we get the effective operators relevant for proton decay

$$
\mathcal{L}_{\text {eff }}=-\frac{g_{5}^{2}}{2 M_{\mathcal{X}}^{2}} \epsilon_{\alpha \beta \gamma} \overline{u_{\alpha, i}^{c}} \gamma^{\mu} Q_{\beta, i}\left[\overline{e_{j}^{c}} \gamma_{\mu} Q_{\gamma, j}-\overline{d_{\gamma, k}^{c}} \gamma_{\mu} L_{k}\right]+\text { h.c. }
$$

With Fierz reordering, one can write the operators as

$$
\mathcal{L}_{\text {eff }}=-\frac{g_{5}^{2}}{M_{\mathcal{X}}^{2}} \epsilon_{\alpha \beta \gamma}\left[\overline{e^{c}} \bar{u}_{\alpha, i}^{c} Q_{\beta, i} Q_{\gamma, j}-\bar{d}_{\alpha, k} \bar{u}_{\beta, i} Q_{\gamma, i} L_{k}\right]+\text { h.c. }
$$


In the case of flipped $\mathrm{SU}(5)$ the $\mathcal{X}^{\prime}=\left(X^{\prime}, Y^{\prime}\right)$ bosons form a $\left(\mathbf{3}^{*}, \mathbf{2},-1 / 6\right)$ representation of $\mathrm{SU}(3) \times \mathrm{SU}(2) \times \mathrm{U}(1)$ with couplings

$$
\mathcal{L}=-i \frac{g_{5}}{\sqrt{2}} \mathcal{X}_{\alpha \mu}^{\prime}\left[\epsilon_{\alpha \beta \gamma} \bar{Q}_{\beta} \gamma^{\mu} d_{\gamma}^{c}-\overline{u^{c}} \gamma^{\mu} L\right]+\text { h.c. } .
$$

Contrary to $\mathrm{SU}(5)$, there is only a single baryon and lepton number violating operator in flipped SU(5),

$$
\mathcal{L}_{\text {eff }}=\frac{g_{5}^{2}}{M_{\mathcal{X}}^{\prime 2}} \epsilon_{\alpha \beta \gamma} \bar{d}_{\alpha, k}^{c} \bar{u}_{\beta, i} Q_{\gamma, i} L_{k}+\text { h.c. }
$$

\subsection{Effective operators in $6 \mathrm{D}$ models}

In the orbifold model described above, the up-type quarks are localised at one fixed point each, in particular the up quark is located at the Georgi-Glashow one. It is therefore clear that dimension-6 proton decay can arise via the exchange of the $\mathrm{SU}(5) X$ and $Y$ bosons as in the traditional $4 \mathrm{D}$ picture. There are though two important differences in the $6 \mathrm{D}$ case, as we will see in the following.

First we have to take into account the presence of not only one $\mathcal{X}$ gauge boson, but of a Kaluza-Klein (KK) tower with masses given by

$$
M_{\mathcal{X}}^{2}(n, m)=\frac{(2 n+1)^{2}}{R_{5}^{2}}+\frac{(2 m)^{2}}{R_{6}^{2}}
$$

for $n, m=0,1,2, \ldots$ The lowest possible mass is $M_{\mathcal{X}}(0,0)=1 / R_{5}$, as given by the $\mathrm{SU}(5)$ breaking parity. Note that if we define the $4 \mathrm{D}$ gauge coupling as the effective coupling of the zero modes, the KK modes interact more strongly by a factor $\sqrt{2}$ due to their bulk normalization.

To obtain the low energy effective operator, we then have to sum over the KK modes,

$$
\begin{aligned}
\frac{1}{\left(M_{\mathcal{X}}^{\text {eff }}\right)^{2}} & =2 \sum_{n, m=0}^{\infty} \frac{1}{M_{\mathcal{X}}^{2}(n, m)} \\
& =2 \sum_{n, m=0}^{\infty} \frac{R_{5}^{2}}{(2 n+1)^{2}+\frac{R_{5}^{2}}{R_{6}^{2}}(2 m)^{2}} .
\end{aligned}
$$

Taking formally the limit $R_{6} / R_{5} \rightarrow 0$, we regain the $5 \mathrm{D}$ result [24],

$$
\frac{1}{\left(M_{\mathcal{X}}^{\mathrm{eff}}\right)^{2}}=2 \sum_{n=0}^{\infty} \frac{R_{5}^{2}}{(2 n+1)^{2}}=\frac{\pi^{2} R_{5}^{2}}{4} .
$$

The double sum in eq. (3.9) is logarithmically divergent. Since our model is valid only below the scale $M_{*}$, where it becomes strongly coupled and also 6D gravity corrections are no longer negligible, we restrict the sum to masses $M_{\mathcal{X}}(n, m) \leq M_{*}$. One easily finds

$$
\frac{1}{\left(M_{\mathcal{X}}^{\mathrm{eff}}\right)^{2}}=\frac{\pi}{4} R_{5} R_{6}\left(\ln \left(M_{*} R_{5}\right)+C\left(\frac{R_{5}}{R_{6}}\right)+\mathcal{O}\left(\frac{1}{R_{5 / 6} M_{*}}\right)\right) .
$$


Note that in the logarithm the smallest KK mass appears, $R_{5}=1 / M_{\mathcal{X}}(0,0)$. The dependence of $1 /\left(M_{\mathcal{X}}^{\text {eff }}\right)^{2}$ on the cutoff $M_{*}$ has to disappear once the model is embedded in a more fundamental theory. In the symmetric case, $R_{5}=R_{6}=1 / M_{c}$, one finds $C(1) \simeq 2.3$ and the expression (3.11) simplifies to

$$
\frac{1}{\left(M_{\mathcal{X}}^{\mathrm{eff}}\right)^{2}} \simeq \frac{\pi}{4 M_{c}^{2}}\left(\ln \left(\frac{M_{*}}{M_{c}}\right)+2.3\right)
$$

Numerically, this agrees with the explicit sum over the KK masses within $1 \%$ for $M_{*} / M_{c}=$ $10 \ldots 50$, which is the relevant range for the ratio of cutoff and compactification scales in $6 \mathrm{D}$.

The second, most important difference of $6 \mathrm{D}$ models compared to $4 \mathrm{D}$ models is the non-universal coupling of the $\mathcal{X}$ gauge bosons. In fact, due to the parities and the $\mathrm{SO}(10)$ breaking pattern, their wavefunctions must vanish on the fixed points with broken $\mathrm{SU}(5)$ symmetry, $O_{\mathrm{PS}}$ and $O_{\mathrm{fl}}$, and therefore no coupling arises via the kinetic term with the charm and top quark or to the brane states $d_{2,3}^{c}, l_{2,3}$. We also have couplings to the bulk states $d_{4}^{c}, d_{4}$ and $l_{4}, l_{4}^{c}$. However, due to the embedding of the zero modes in full $\mathrm{SU}(5)$ multiplets together with massive KK modes, i.e. $\left(d_{4}^{c}, L_{4}\right),\left(d_{4}, L_{4}^{c}\right),\left(D_{4}^{c}, l_{4}\right)$ and $\left(D_{4}, l_{4}^{c}\right)$, the charged current interaction always mixes the light states with the heavy ones, and it is therefore irrelevant for the low energy process of proton decay [24]. So the kinetic coupling in eq. (3.5) arises only for a single flavour eigenstate, not for all flavours as in the usual 4D case.

\subsection{Corrections from derivative brane operators}

Apart from the kinetic term couplings, at any brane additional couplings can arise containing derivatives along the extra dimensions of the locally vanishing gauge bosons. Such operators are a $6 \mathrm{D}$ generalization of the $5 \mathrm{D}$ derivative operators discussed in [24, 34 ,

$$
\mathcal{L}_{d}=\sum_{\text {fixed points }} \delta_{i}(z) \frac{c_{5 / 6}^{i}}{M_{*}} \int_{\theta^{2} \bar{\theta}^{2}} \bar{\Phi}_{1}\left(\mathcal{D}_{5 / 6} e^{2 V}\right) \Phi_{2}+\text { h.c. }
$$

Here $c_{5 / 6}^{i}$ are unknown brane coefficients, $\mathcal{D}_{5 / 6}=\partial_{5 / 6}+i A_{5 / 6}$ are the covariant derivatives in the extra dimensions and $\Phi_{i}$ are any two different, locally non-vanishing fields in group representations which form a singlet together with generators of the broken symmetries. For unbroken symmetries the chiral superfields $A_{5 / 6}$ vanish at the fixpoints.

These supersymmetric terms produce on the GG brane couplings with the flipped $\mathrm{SU}(5)$ leptoquark gauge bosons $\mathcal{X}^{\prime}$, whose derivatives do not vanish on that brane. On the flipped SU(5) brane, there are couplings containing the derivative of the $\mathcal{X}$ gauge bosons, and on the Pati-Salam brane there are derivative couplings with both $\mathcal{X}^{\prime}$ and $\mathcal{X}$. Due to these additional vertices, three different classes of operators can arise:

- operators coming from $\mathcal{X}^{\prime}$ exchange on the GG brane: these involve two derivative vertices and can produce additional contributions to the effective operator

$$
\overline{d_{k}^{c}} \overline{u_{1}^{c}} Q_{1} L_{j}
$$

with $k, j=1,4$; we will discuss their contribution below; 
- operators coming from $\mathcal{X}$-exchange on the flipped $\mathrm{SU}(5)$ brane or $\mathcal{X}, \mathcal{X}^{\prime}$ exchange on the Pati-Salam brane: these usually involve the charm and top quark instead of the up quark, and they are therefore irrelevant for proton decay;

- interbrane operators from both the exchanges of $\mathcal{X}$ and $\mathcal{X}^{\prime}$ gauge bosons: they can involve either one or two derivative vertices and generate mixed flavour operators of the type

$$
\begin{aligned}
& \overline{e_{j}^{c}} \overline{u_{1}^{c}} u_{1} d_{k}-\overline{d_{k}^{c}} \overline{u_{1}^{c}} Q_{1} L_{j} \quad k, j=2,3,4 \quad \mathcal{X} \text { exchange, } \\
& \overline{d_{2}^{c}} \overline{u_{1}^{c}} d_{2} \nu_{k} \quad k=1,4 \quad \mathcal{X}^{\prime} \text { exchange GG-fl, } \\
& \overline{d_{j}^{c}} \overline{u_{1}^{c}} d_{l} \nu_{k} \quad j, l=3,4 ; k=1,4 \quad \mathcal{X}^{\prime} \text { exchange GG-PS. }
\end{aligned}
$$

Apart from the last term, they are usually suppressed compared to the kinetic term operators by a factor $M_{c} / M_{*}$ due to the different parities of the vertices.

To estimate the effect of these additional operators, which introduce a dependence on the $\mathcal{O}(1)$ coefficients $c_{5 / 6}$, let us now consider the KK summations with one or two derivative vertices. We restrict ourselves here to the case $R_{5}=R_{6}=1 / M_{c}$ and universal coefficients $c_{5 / 6}$ at the different fixpoints. Note that even if suppressed by $M_{*}$, these operators can be as important as the usual ones, since the derivative enhances the divergence of the KK summation, which compensates the suppression. For example, from the exchange of the $\mathcal{X}^{\prime}$ bosons on the GG brane, we obtain the sum

$$
\frac{1}{\left(M_{\mathcal{X}}^{\text {eff }}\right)_{\text {b.o. }}^{2}}=\frac{2}{M_{*}^{2}} \sum_{n, m} \frac{\left|c_{5}(2 n+1)+c_{6}(2 m+1)\right|^{2}}{(2 n+1)^{2}+(2 m+1)^{2}},
$$

which is quadratically divergent. Using again the cutoff $M_{\mathcal{X}}(n, m) \leq M_{*}$, we obtain

$$
\frac{1}{\left(M_{\mathcal{X}}^{\text {eff }}\right)_{\text {b.o. }}^{2}}=\frac{\pi}{16 M_{c}^{2}}\left(\left|c_{5}\right|^{2}+\left|c_{6}\right|^{2}+\frac{4}{\pi} \operatorname{Re}\left[c_{5} c_{6}^{*}\right]+\mathcal{O}\left(\frac{M_{c}}{M_{*}}\right)\right),
$$

to be compared with the 'canonical' term eq. (3.12). A similar result is obtained for the exchange of $\mathcal{X}^{\prime}$ gauge bosons between the GG and the PS brane, affecting only the decay into neutrinos.

The exchange of gauge bosons between different branes involving only one single derivative operator are less dangerous since the propagator gives a factor $(-1)^{n}$ accounting for the different parities of the derivative and the kinetic term vertices. Therefore the KK summations are in general suppressed by a factor $M_{c} / M_{*}$. The exchange of $\mathcal{X}$ bosons between the GG and the flipped $\mathrm{SU}(5)$ brane gives

$$
\frac{1}{\left(M_{\mathcal{X}}^{\text {eff }}\right)_{\text {b.o. }}^{2}}=\frac{2}{M_{c} M_{*}} \sum_{n, m}(-1)^{n} \frac{c_{5}(2 n+1)+c_{6}(2 m)}{(2 n+1)^{2}+(2 m)^{2}} .
$$

This sum is only logarithmically divergent thanks to the alternating signs. Using again the cutoff $M_{\mathcal{X}}(n, m) \leq M_{*}$, one finds

$$
\frac{1}{\left(M_{\mathcal{X}}^{\text {eff }}\right)_{\text {b.o. }}^{2}}=\frac{1}{2 M_{c} M_{*}}\left(c_{6} \ln \left(\frac{M_{*}}{M_{c}}\right)+\mathcal{O}(1)\right),
$$

which is suppressed compared to the 'canonical' term by a factor $M_{c} / M_{*}$. The same result is obtained for the $\mathcal{X}^{\prime}$ exchange between the GG and the flipped $\mathrm{SU}(5)$ branes. 
Note that in principle operators with a higher number of derivatives can also be present, which contribute at the same level as the single derivative ones because the divergence of the KK summation compensates the suppression by powers of $M_{*}$. We will assume that their contribution is small.

Regarding the $\mathrm{N}=2$ scalar superpartners of the $\mathrm{SU}(5)$ gauge bosons, $\mathcal{X}_{5,6}$, the brane terms in eq. (3.13) give rise to couplings with fermion kinetic terms which can only produce corrections of order $\left(m_{p} / M_{*}\right)^{2}$. The derivatives of the $\mathcal{X}_{5,6}$ bosons do not couple to fermion pairs and are therefore irrelevant.

Finally, we emphasize that the position of the lightest quark generation is crucial for the discussion of proton decay. For instance, if the up quark were located on the Pati-Salam brane, the dimension- 6 operator coming from the kinetic terms would be absent since both the $\mathcal{X}$ bosons of $\mathrm{SU}(5)$ and the $\mathcal{X}^{\prime}$ bosons of flipped $\mathrm{SU}(5)$ vanish there. In principle, this gives us a means to avoid the 'canonical' dimension- 6 operators completely, leaving the derivative couplings as dominant contributions.

\section{Flavour structure and branching ratios}

\subsection{Flavour mixing in $6 \mathrm{D}$ versus $4 \mathrm{D}$ GUT models}

Proton decay involves only the light quark states and the operators containing the combinations $u u d$ and $u d d$. Therefore we have to rotate the weak eigenstates into the mass eigenstates and single out the contributions for the lightest generation. Without loss of generality and for future convenience, we can start in the basis where the up-quark mass matrix is diagonal. Then the down quark and lepton mass matrices are not diagonal, in general, but can be diagonalised by unitary transformations,

$$
\begin{aligned}
& d_{L}=U_{L}^{d} d_{L}^{\prime}, \quad e_{L}=U_{L}^{e} e_{L}^{\prime}, \quad \nu_{L}=U_{L}^{\nu} \nu_{L}^{\prime}, \\
& d_{R}=U_{R}^{d} d_{R}^{\prime}, \quad e_{R}=U_{R}^{e} e_{R}^{\prime},
\end{aligned}
$$

where the prime denotes mass eigenstates. Since the up-quark matrix is diagonal, $U_{L}^{d}$ coincides with the CKM matrix.

We can now express the proton decay operators of eq. (3.5) in term of mass eigenstates,

$$
\begin{aligned}
\mathcal{L}_{\text {eff }}=\frac{g_{5}^{2}}{M_{\mathcal{X}}^{2}} \epsilon_{\alpha \beta \gamma}[ & {\overline{e_{k}^{c}}}_{k}^{\prime}\left(U_{R}^{e \top}\right)_{k j} \overline{u_{\alpha, i}^{c}}\left(d_{\beta, m}^{\prime}\left(U_{L}^{d}\right)_{i m} u_{\gamma, j}-u_{\beta, i}\left(U_{L}^{d}\right)_{j l} d_{\gamma, l}^{\prime}\right)+ \\
& \left.+{\overline{d^{c}}}_{\alpha, l}^{\prime}\left(U_{R}^{d \top}\right)_{l k} \overline{u^{c}}{ }_{\beta, i}\left(u_{\gamma, i}\left(U_{L}^{e}\right)_{k j} e_{j}^{\prime}-d_{\gamma, m}^{\prime}\left(U_{L}^{d}\right)_{i m}\left(U_{L}^{\nu}\right)_{k j} \nu_{j}^{\prime}\right)\right]+ \text { h.c. }
\end{aligned}
$$

Note that for the orbifold construction, where only the first generation weak eigenstates couple to the $\mathcal{X}$ bosons, the effective operators read instead

$$
\begin{aligned}
\mathcal{L}_{\text {eff }}=\frac{g_{5}^{2}}{\left(M_{\mathcal{X}}^{\text {eff }}\right)^{2}} \epsilon_{\alpha \beta \gamma}[ & 2{\overline{e_{k}^{\prime}}}_{k}^{\prime}\left(U_{R}^{e \top}\right)_{k 1} \overline{u_{\alpha, 1}^{c}} d_{\beta, m}^{\prime}\left(U_{L}^{d}\right)_{1 m} u_{\gamma, 1}+ \\
& \left.+{\overline{d^{c}}}_{\alpha, l}^{\prime}\left(U_{R}^{d \top}\right)_{l 1} \overline{u^{c}}{ }_{\beta, 1}\left(u_{\gamma, 1}\left(U_{L}^{e}\right)_{1 j} e_{j}^{\prime}-d_{\gamma, m}^{\prime}\left(U_{L}^{d}\right)_{1 m}\left(U_{L}^{\nu}\right)_{1 j} \nu_{j}^{\prime}\right)\right]+ \text { h.c. }
\end{aligned}
$$


Let us analyze the mixing pattern for the down quarks in the orbifold model. Since the mass matrices of down quarks and charged leptons both have the form eq. (2.4), $m^{d} \sim m^{e} \sim m, U_{R}^{d}$ and $U_{L}^{e}$ have the same structure but, in general, coefficients $\mathcal{O}(1)$ will be different. For $\mu_{1}, \mu_{2} \ll \mu_{3}$, we obtain for the right-handed down-type quarks and left-handed charged leptons,

$$
U_{R}^{d} \sim U_{L}^{e} \sim V_{4} V_{3}=\left(\begin{array}{cccc}
-\frac{\widetilde{M}_{2}}{\widetilde{M}_{12}} \frac{\widetilde{M}_{1}\left(\widetilde{\mu}_{3} \widetilde{M}_{3}-\mu_{3} \widetilde{M}_{4}\right)}{\bar{\mu}_{3} \widetilde{M}_{12}} & -\frac{\widetilde{M}_{1}\left(\widetilde{\mu}_{3} \widetilde{M}_{4}+\mu_{3} \widetilde{M}_{3}\right)}{\bar{\mu}_{3} \widetilde{M}^{2}} & \frac{\widetilde{M}_{1}}{\bar{M}} \\
\frac{\widetilde{M}_{1}}{\widetilde{M}_{12}} \frac{\widetilde{M}_{2}\left(\widetilde{\mu}_{3} \widetilde{M}_{3}-\mu_{3} \widetilde{M}_{4}\right)}{\bar{\mu}_{3} \widetilde{M} \widetilde{M}_{12}} & -\frac{\widetilde{M}_{2}\left(\widetilde{\mu}_{3} \widetilde{M}_{4}+\mu_{3} \widetilde{M}_{3}\right)}{\bar{\mu}_{3} \widetilde{M}^{2}} & \frac{\widetilde{M}_{2}}{\bar{M}} \\
0 & -\frac{\widetilde{\mu}_{3}}{\bar{\mu}_{3} \frac{\widetilde{M}_{12}}{M}} & -\frac{\widetilde{\mu}_{3} \widetilde{M}_{3} \widetilde{M}_{4}-\mu_{3}\left(\widetilde{M}_{1}^{2}+\widetilde{M}_{2}^{2}+\widetilde{M}_{4}^{2}\right)}{\bar{\mu}_{3} \widetilde{M}^{2}} & \frac{\widetilde{M}_{3}}{\bar{M}} \\
0 & \frac{\mu_{3}}{\bar{\mu}_{3} \frac{\widetilde{M}_{12}}{M}} & \frac{\widetilde{\mu}_{3}\left(\widetilde{M}_{1}^{2}+\widetilde{M}_{2}^{2}+\widetilde{M}_{3}^{2}\right)-\mu_{3} \widetilde{M}_{3} \widetilde{M}_{4}}{\bar{\mu}_{3} \widetilde{M}^{2}} & \frac{\widetilde{M}_{4}}{\bar{M}}
\end{array}\right),
$$

up to the two-dimensional mixing matrix for the second and third generation discussed in section 2 .

The rotation matrices of the left-handed down quarks and right-handed leptons are obtained by diagonalizing $m^{\top} m$, which leads to small mixing angles, with

$$
U_{L}^{d}=V_{\mathrm{CKM}} \sim U_{R}^{e}
$$

The unitary matrices $U_{L}^{e}, U_{R}^{d}, U_{L}^{d}$ and $U_{R}^{e}$ determine the coefficients of the proton decay operators in eq. (4.4).

To make a comparison with ordinary 4D GUT models, we consider the flavour structure of two SU(5) models described in refs. [35, 36]. These models make use of the FroggattNielsen mechanism [37] where a global $\mathrm{U}(1)_{F}$ flavour symmetry is broken spontaneously by the VEV of gauge singlet field $\Phi$ at a high scale. Then the Yukawa couplings arise from the non-renormalizable operators,

$$
h_{i j}=g_{i j}\left(\frac{\langle\Phi\rangle}{\Lambda}\right)^{Q_{i}+Q_{j}} .
$$

Here, $g_{i j}$ are couplings $\mathcal{O}(1)$ and $Q_{i}$ are the $\mathrm{U}(1)_{F}$ charges of the various fermions. Particularly interesting is the case with a 'lopsided' family structure, where the chiral charges are different for $\mathbf{5}^{*}$ and $\mathbf{1 0}$ of the same family. The two examples [35, 36] with phenomenologically allowed lopsided charges are given in table 1 .

Note that in these models the large neutrino mixing is explained by a large mixing of $\mathbf{5}^{*}$-plets, which is analogous to the large mixing of lepton doublets and right-handed down quarks in the $6 \mathrm{D}$ model described above. Contrary to the $6 \mathrm{D}$ model, this does not determine the $\mathrm{U}(1)_{F}$ charges of the right-handed neutrinos. For proper choices these models also lead to successful baryogenesis via leptogenesis [38.

The charge assignments determine the structure of the Yukawa matrices. In model A [35], corresponding to the semi-anarchical model of [36], the couplings for down quarks 


\begin{tabular}{|c|ccc|ccc|}
\hline$Q_{F}$ & $\mathbf{1 0}_{3}$ & $\mathbf{1 0}_{2}$ & $\mathbf{1 0}_{1}$ & $\mathbf{5}_{3}^{*}$ & $\mathbf{5}_{2}^{*}$ & $\mathbf{5}_{1}^{*}$ \\
\hline model A & 0 & 1 & 2 & $a$ & $a$ & $a+1$ \\
model B & 0 & 3 & 5 & 0 & 0 & 2 \\
\hline
\end{tabular}

Table 1: $\mathrm{U}(1)_{F}$ charges of the $\mathrm{SU}(5)$ fields; $a=0,1$.

and charged leptons read

$$
h_{d} \sim h_{e} \sim \epsilon^{a}\left(\begin{array}{ccc}
\epsilon^{3} & \epsilon^{2} & \epsilon^{2} \\
\epsilon^{2} & \epsilon & \epsilon \\
\epsilon & 1 & 1
\end{array}\right),
$$

where the parameter $\epsilon=\langle\Phi\rangle / \Lambda \sim 1 / 17$ controls the flavour mixing. Diagonalisation of the Yukawa matrices yields

$$
U_{L}^{d}=V_{\mathrm{CKM}} \sim U_{R}^{e} \sim\left(\begin{array}{ccc}
1 & \epsilon & \epsilon^{2} \\
\epsilon & 1 & \epsilon \\
\epsilon^{2} & \epsilon & 1
\end{array}\right), \quad U_{R}^{d} \sim U_{L}^{e} \sim\left(\begin{array}{ccc}
1 & \epsilon & \epsilon \\
\epsilon & 1 & 1 \\
\epsilon & 1 & 1
\end{array}\right) .
$$

In model B, which corresponds to the hierarchical $H_{I I}$ model of 36], the structure is similar, while the small parameter is instead $\lambda \sim 0.35$, such that $\lambda^{2} \sim \epsilon$.

\subsection{Decay rates and branching ratios}

To calculate the decay rates, we have to evaluate the hadron matrix elements $\langle P S|\mathcal{O}| p\rangle$, which describe the transition from the proton via the three-quark operator $\mathcal{O}$ to a pseudo scalar meson. The various matrix elements are calculated from the basic element

$$
\alpha P_{L} u_{p}=\epsilon_{\alpha \beta \gamma}\left\langle 0\left|\left(d_{R}^{\alpha} u_{R}^{\beta}\right) u_{L}^{\gamma}\right| p\right\rangle
$$

with the aid of chiral perturbation theory [39, 40]; $u_{p}$ denotes the proton spinor. The decay rates for the different channels are given in table 2. Here $m_{p}, m_{\pi}, m_{K}$ and $m_{\eta}$ denote the masses of proton, pion, kaon and eta, respectively, and $f_{\pi}$ is the pion decay constant; $m_{B}=1.15 \mathrm{GeV}$ is an average baryon mass according to contributions from diagrams with virtual $\Sigma$ and $\Lambda ; D=0.80$ and $F=0.46$ are the symmetric and antisymmetric $\mathrm{SU}(3)$ reduced matrix elements for the axial-vector current [41. The matrix element $\alpha$ is evaluated by means of lattice QCD simulations; its absolute value varies in the range $(0.003-0.03) \mathrm{GeV}^{3}$. We will choose $|\alpha|=0.01 \mathrm{GeV}^{3}$ (see the recent discussion in Ref. [42]).

In the decay rates listed in Table 2 the coupling constant $G_{G}=g_{5}^{2} /\left(M_{\mathcal{X}}^{\text {eff }}\right)^{2}$ is given by eqs. (3.12), (3.19) and (3.21). The operators have to be evolved from the GUT scale down to the hadronic scale, which is described by the factor $A=A^{\mathrm{SD}} \cdot A^{\mathrm{LD}}$. It contains both a short-distance contribution $A^{\mathrm{SD}}=2.37$, for the evolution from the GUT scale to the SUSY-breaking scale, and a long-distance contribution $A^{\mathrm{LD}}=1.43$, for the evolution from the SUSY-breaking scale to $1 \mathrm{GeV}$ [13. The effect of lepton masses is neglected.

The quark and lepton mixing patterns discussed above fix the coefficients $C_{i j k m}^{2}$ in the decay rates. As an example, for the process $p \rightarrow e^{+} \pi^{0}$, we obtain from eq. (4.4),

$$
C_{u d u e}^{2}=4\left[\left(U_{R}^{e}\right)_{11}\left(U_{L}^{d}\right)_{11}\right]^{2}+\left[\left(U_{R}^{d}\right)_{11}\left(U_{L}^{e}\right)_{11}\right]^{2} .
$$




$$
\begin{aligned}
\Gamma\left(p \rightarrow e_{j}^{+} \pi^{0}\right) & =\frac{\left(m_{p}^{2}-m_{\pi^{0}}^{2}\right)^{2}}{32 \pi m_{p}^{3} f_{\pi}^{2}} \alpha^{2} A^{2} G_{G}^{2}\left(\frac{1+D+F}{\sqrt{2}}\right)^{2} C_{u d u e_{j}}^{2} \\
\Gamma\left(p \rightarrow \bar{\nu}_{j} \pi^{+}\right) & =\frac{\left(m_{p}^{2}-m_{\pi \pm}^{2}\right)^{2}}{32 \pi m_{p}^{3} f_{\pi}^{2}} \alpha^{2} A^{2} G_{G}^{2}(1+D+F)^{2} C_{u d d \nu_{j}}^{2} \\
\Gamma\left(p \rightarrow e_{j}^{+} K^{0}\right) & =\frac{\left(m_{p}^{2}-m_{K^{0}}^{2}\right)^{2}}{32 \pi m_{p}^{3} f_{\pi}^{2}} \alpha^{2} A^{2} G_{G}^{2}\left(1+(D-F) \frac{m_{p}}{m_{B}}\right)^{2} C_{u s u e_{j}}^{2} \\
\Gamma\left(p \rightarrow \bar{\nu}_{j} K^{+}\right) & =\frac{\left(m_{p}^{2}-m_{K \pm}^{2}\right)^{2}}{32 \pi m_{p}^{3} f_{\pi}^{2}} \alpha^{2} A^{2} G_{G}^{2}\left[\left(\frac{2}{3} D \frac{m_{p}}{m_{B}}\right) C_{u s d \nu_{j}}+\left(1+\frac{D+3 F}{3} \frac{m_{p}}{m_{B}}\right) C_{u d s \nu_{j}}\right]^{2} \\
\Gamma\left(p \rightarrow e_{j}^{+} \eta\right) & =\frac{\left(m_{p}^{2}-m_{\eta}^{2}\right)^{2}}{32 \pi m_{p}^{3} f_{\pi}^{2}} \alpha^{2} A^{2} G_{G}^{2}\left(\frac{1+D-3 F}{\sqrt{6}}\right)^{2} C_{u d u e_{j}}^{2}
\end{aligned}
$$

Table 2: Partial widths of proton decay channels [43].

The coefficients for the other processes can be read off analogously. The decay rates in table 2 have the same form as the decay rates determined by dimension- 5 operators. The difference lies in the coefficients $C_{i j k m}$ and the coupling constant $G_{G}$.

We now start with the simplest case of our orbifold model, with $U_{R}^{d}=U_{L}^{e}$, and with degenerate masses $\widetilde{M}$ and $\widetilde{\mu}_{3}=\mu_{3}$, which we denote as case I. The mixing matrix for righthanded down quarks and left-handed charged leptons, eq. (4.5), is then simply given by

$$
U_{R}^{d}=U_{L}^{e}=\left(\begin{array}{cccc}
-\frac{1}{\sqrt{2}} & 0 & -\frac{1}{2} & \frac{1}{2} \\
\frac{1}{\sqrt{2}} & 0 & -\frac{1}{2} & \frac{1}{2} \\
0 & -\frac{1}{\sqrt{2}} & \frac{1}{2} & \frac{1}{2} \\
0 & \frac{1}{\sqrt{2}} & \frac{1}{2} & \frac{1}{2}
\end{array}\right),
$$

thus the state $d_{1}^{c}$ has no strange-component. For the current-current operators we then obtain from eq. (4.4),

$$
\begin{aligned}
\mathcal{L}_{\text {eff }} \simeq \frac{g_{5}^{2}}{\left(M_{\mathcal{X}}^{\text {eff }}\right)^{2}} \epsilon_{\alpha \beta \gamma}[ & 2 V_{u d}^{2} \overline{e^{c}} \overline{u_{\alpha}^{c}} d_{\beta} u_{\gamma}+\frac{1}{2} \overline{d_{\alpha}^{c}} \overline{u_{\beta}^{c}} u_{\gamma} e+2 V_{u d} V_{u s} \overline{\mu^{c}} \overline{u_{\alpha}^{c}} d_{\beta} u_{\gamma}+ \\
& +2 V_{u d} V_{u s} \overline{e^{c}} \overline{u_{\alpha}^{c}} s_{\beta} u_{\gamma}+2 V_{u s}^{2} \overline{\mu^{c}} \overline{u_{\alpha}^{c}} s_{\beta} u_{\gamma}- \\
& \left.-\sum_{j=1}^{3} \frac{1}{\sqrt{2}}\left(U_{L}^{\nu}\right)_{1 j} \overline{u_{\alpha}^{c}} \overline{d_{\beta}^{c}}\left\{V_{u d} d_{\gamma}+V_{u s} s_{\gamma}\right\} \nu_{j}\right]+ \text { h.c. },
\end{aligned}
$$

where the fermions are now mass eigenstates. From this equation we can read off the coefficients of the various coefficients $C_{i j k m}$ appearing in the decay rates (cf. table 2),

$$
\begin{aligned}
& C_{u d u e}^{2}=4 V_{u d}^{4}+\frac{1}{4}, \quad C_{u s u \mu}^{2}=4 V_{u s}^{4}, \quad C_{u d u \mu}^{2}=C_{u s u e}^{2}=4 V_{u s}^{2} V_{u d}^{2}, \\
& C_{u d d \nu}^{2}=\frac{1}{2} V_{u d}^{2}, \quad C_{u d s \nu}=\frac{1}{\sqrt{2}} V_{u s}, \quad C_{u s d \nu}=0,
\end{aligned}
$$

where we have used $\sum_{j=1}^{3}\left(U_{L}^{\nu}\right)_{1 j}\left(U_{L}^{\nu}\right)_{1 j}^{*}=1$.

The numerical results for the branching ratios are listed in table 3. Note that the effect of the derivative operators is negligible for $c_{5}=c_{6}=1$. For the listed branching ratios the corrections are less than $3 \%$. 


\begin{tabular}{|c|cc|c|}
\hline decay channel & \multicolumn{3}{|c|}{ Branching Ratios [\%] } \\
\cline { 2 - 4 } & \multicolumn{2}{|c|}{ 6D SO $(10)$} & $\mathrm{SU}(5) \times \mathrm{U}(1)_{F}$ \\
& case I & case II & models A \& B \\
\hline$e^{+} \pi^{0}$ & 75 & 71 & 54 \\
$\mu^{+} \pi^{0}$ & 4 & 5 & $<1$ \\
$\bar{\nu} \pi^{+}$ & 19 & 23 & 27 \\
$e^{+} K^{0}$ & 1 & 1 & $<1$ \\
$\mu^{+} K^{0}$ & $<1$ & $<1$ & 18 \\
$\bar{\nu} K^{+}$ & $<1$ & $<1$ & $<1$ \\
$e^{+} \eta$ & $<1$ & $<1$ & $<1$ \\
$\mu^{+} \eta$ & $<1$ & $<1$ & $<1$ \\
\hline
\end{tabular}

Table 3: Resulting branching ratios and comparision with $\mathrm{SU}(5) \times \mathrm{U}(1)_{F}$.

To compare the branching ratios of the $6 \mathrm{D}$ model with those of the two $4 \mathrm{D}$ GUT models described above, we assume that some mechanism suppresses or avoids the proton decay arising from dimension-5 operators. The coefficients $C_{i j k l}^{2}$ can then be derived from eq. (4.3) using the mixing matrices given in eq. (4.9). For model A, they read

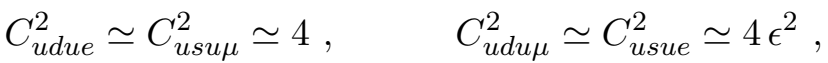

$$
\begin{aligned}
& C_{u d d \nu}^{2} \simeq \frac{1}{2}, \quad C_{u d s \nu} \simeq \frac{1}{\sqrt{2}}, \quad C_{u s d \nu} \simeq 2 \epsilon .
\end{aligned}
$$

In model $\mathrm{B}, \epsilon$ is replaced by $\lambda^{2}$. The differences between the branching ratios of the two models are not significant.

The difference between the $6 \mathrm{D} \mathrm{SO}(10)$ and the $4 \mathrm{D} \mathrm{SU}(5)$ models is most noticeable in the channel $p \rightarrow \mu^{+} K^{0}$. This is due to the absence of second and third generation weak eigenstates in the current-current operators and the vanishing (12)-component in $U_{R}^{d}$ and $U_{L}^{e}$ in the case of the $6 \mathrm{D}$ model. Hence, the decay $p \rightarrow \mu^{+} K^{0}$ is doubly Cabibbo suppressed. This effect is a direct consequence of the localisation of the 'first generation' to the Georgi-Glashow brane.

Let us now consider the general case, where the $\widetilde{M}^{(d, e)}$ are not degenerate, and where $\mu_{3}$ and $\widetilde{\mu}_{3}^{(d, e)}$ differ as well. From eq. (4.5) we see that the strange component in $d_{1}^{c}$ does not vanish anymore, but it is smaller than the bottom component. We have studied several cases whose results agree remarkably well. As an illustration, consider the case where $\widetilde{\mu}_{3}^{d}=$ $2 \mu_{3}$ and $\widetilde{\mu}_{3}^{e}=3 \mu_{3}$, with non-degenerate heavy masses $\widetilde{M}_{1}^{d}: \widetilde{M}_{2}^{d}: \widetilde{M}_{3}^{d}: \widetilde{M}_{4}^{d}=\frac{1}{2}: \frac{1}{\sqrt{2}}: \frac{1}{\sqrt{2}}: 1$ and $\widetilde{M}_{1}^{e}: \widetilde{M}_{2}^{e}: \widetilde{M}_{3}^{e}: \widetilde{M}_{4}^{e}=\frac{1}{2}: \frac{1}{\sqrt{2}}: 1: \frac{1}{2}$ (case II). The branching ratios are listed in table 3 ; the differences between the two cases are indeed small.

The most striking difference is the decay channel $p \rightarrow \mu^{+} K^{0}$, which is suppressed by about two orders of magnitude in the $6 \mathrm{D}$ model with respect to $4 \mathrm{D}$ models. It is therefore important to determine an upper limit for this channel in the $6 \mathrm{D}$ model. Varying the mass parameters in the range $\widetilde{M}_{j} / \widetilde{M}=0.1-1$ and $\widetilde{\mu}_{3}^{d, e} / \mu_{3}=0.1-10$, we find

$$
\frac{\Gamma\left(p \rightarrow \mu^{+} K^{0}\right)}{\Gamma\left(p \rightarrow e^{+} \pi^{0}\right)} \lesssim 5 \% \text {. }
$$


Finally, a limit on the compactification scale can be derived from the decay width of the dominant channel $p \rightarrow e^{+} \pi^{0}$. Neglecting the suppressed contributions from the derivative operators, we obtain the analytic expression

$$
\begin{aligned}
\Gamma\left(p \rightarrow e^{+} \pi^{0}\right) \simeq & \frac{\left(m_{p}^{2}-m_{\pi^{0}}^{2}\right)^{2}}{32 \pi m_{p}^{3} f_{\pi}^{2}} \alpha^{2} A^{2}\left(\frac{1+D+F}{\sqrt{2}}\right)^{2} \times \\
& \times \frac{\pi^{2}}{16 M_{c}^{4}}\left(\ln \left(\frac{M_{*}}{M_{c}}\right)+2.3\right)^{2}\left[4 V_{u d}^{4}+\frac{\widetilde{M}_{2}^{d 2}}{\widetilde{M}_{1}^{d 2}+\widetilde{M}_{2}^{d 2}} \frac{\widetilde{M}_{2}^{e 2}}{\widetilde{M}_{1}^{e 2}+\widetilde{M}_{2}^{e 2}}\right] \\
\simeq & \left(\frac{9 \times 10^{15} \mathrm{GeV}}{M_{c}}\right)^{4}\left(\frac{\alpha}{0.01 \mathrm{GeV}^{3}}\right)^{2}\left(5.3 \times 10^{33} \mathrm{yrs}\right)^{-1} .
\end{aligned}
$$

With $M_{*}=10^{17} \mathrm{GeV}$ and $\widetilde{M}_{1,2}^{d, e}=\mathcal{O}(1)$, the experimental limit $\tau \geq 5.3 \times 10^{33}$ yields $M_{c} \geq M_{c}^{\text {min }} \simeq 9 \times 10^{15} \mathrm{GeV}$, which is very close to the $4 \mathrm{D}$ GUT scale. The lower bound corresponds to $M_{*} / M_{c}^{\min }=12$ and $\left(M_{\chi}^{\text {eff }}\right)^{2} /\left(M_{c}^{\min }\right)^{2}=3.72$.

\section{Conclusions}

We have studied proton decay in a $6 \mathrm{D} \mathrm{SO}(10)$ orbifold GUT model, which is determined by dimension- 6 operators. The characteristic features of the model are the different breakings of the $\mathrm{SO}(10)$ symmetry at different points in the extra dimensions and the associated localisation of some quarks and leptons. We find that, like in 5D orbifold GUTs, the proton decay rate is enhanced and the branching ratios are strongly affected by the quark-lepton 'geography' [23, 24].

The summation over Kaluza-Klein towers depends logarithmically on the cutoff scale $M_{*}$ in $6 \mathrm{D}$, contrary to $5 \mathrm{D}$, where the sum is finite. Identifying the cutoff with the $6 \mathrm{D}$ Planck mass, i.e. $M_{*} \simeq 10^{17} \mathrm{GeV}$, the SuperKamiokande bound on the proton lifetime leads to the lower bound on the compactification scale $M_{c}>9 \times 10^{15} \mathrm{GeV}$. On the other hand, the approximate unification of gauge couplings suggests $M_{c} \simeq \Lambda_{\mathrm{GUT}} \simeq 2 \times 10^{16} \mathrm{GeV}$. This yields the proton lifetime $\tau\left(p \rightarrow e^{+} \pi^{0}\right) \simeq 1 \times 10^{35}$ yrs which, remarkably, lies within the reach of the next generation of large volume detectors!

The peculiar flavour structure of our orbifold GUT model leads to characteristic signatures in the branching ratios of proton decay, in particular the strong suppression of the mode $p \rightarrow \mu^{+} K^{0}$ compared to the predictions of $4 \mathrm{D}$ models. The reason is the higher-dimensional quark-lepton 'geography' and the related non-universal couplings of GUT bosons to fermions. Such a pattern can be tested already with a handful of events! Hence, the discovery of proton decay may not only confirm the most striking prediction of grand unification, but it might also reveal its higher dimensional origin.

\section{Acknowledgments}

We would like to thank A. Hebecker for helpful discussions. L.C. would also like to thank J. Ellis, and A. Romanino, C. Scrucca and the other participants of the CERN Phen Club on 26/2/2004 for useful discussions and suggestions. The work of D.E.C. was supported by Fundação para a Ciência e a Tecnologia under the grant SFRH/BPD/1598/2000. 


\section{References}

[1] J.C. Pati and A. Salam, Lepton number as the fourth color, Phys. Rev. D 10 (1974) 275.

[2] H. Georgi and S.L. Glashow, Unity of all elementary particle forces, Phys. Rev. Lett. 32 (1974) 438.

[3] H. Georgi, Particles and Fields 1974, C.E. Carlson ed., AIP, NY, 1975, p. 575.

[4] H. Fritzsch and P. Minkowski, Unified interactions of leptons and hadrons, Ann. Phys. (NY) $93(1975) 193$.

[5] S.M. Barr, A new symmetry breaking pattern for $\mathrm{SO}(10)$ and proton decay, Phys. Lett. B 112 (1982) 219.

[6] J.P. Derendinger, J.E. Kim and D.V. Nanopoulos, Anti - SU(5), Phys. Lett. B 139 (1984) 170 .

[7] TITAND Working Group collaboration, Y. Suzuki et al., Multi-megaton water cherenkov detector for a proton decay search: titand (former name: titanic), hep-ex/0110005.

[8] Super-Kamiokande Collaboration, K. S. Ganezer et al., The search for nucleon decay at Super-Kamiokande, Int. J. Mod. Phys. A 16S1B (2001) 855.

[9] T. Goto and T. Nihei, Effect of rrrr dimension 5 operator on the proton decay in the minimal SU(5) sugra GUT model, Phys. Rev. D 59 (1999) 115009 hep-ph/9808255.

[10] R. Dermisek, A. Mafi, S. Raby, SUSY GUTs under siege: proton decay, Phys. Rev. D 63 (2001) 035001 hep-ph/0007213.

[11] H. Murayama and A. Pierce, Not even decoupling can save minimal supersymmetric SU(5), Phys. Rev. D 65 (2002) 055009 hep-ph/0108104.

[12] B. Bajc, P.F. Perez and G. Senjanović, Proton decay in minimal supersymmetric SU(5), Phys. Rev. D 66 (2002) 075005 hep-ph/0204311.

[13] D. Emmanuel-Costa and S. Wiesenfeldt, Proton decay in a consistent supersymmetric SU(5) GUT model, Nucl. Phys. B 661 (2003) 62 hep-ph/0302272.

[14] H.S. Goh, R.N. Mohapatra, S. Nasri and S.-P. Ng, Proton decay in a minimal SUSY SO(10) model for neutrino mixings, Phys. Lett. B 587 (2004) 105 hep-ph/0311330.

[15] C. Jarlskog, Inherent mixings in the grand unified $\mathrm{SU}(5)$ model and proton lifetime, Phys. Lett. B 82 (1979) 401.

[16] R.N. Mohapatra, Flavor mixing in SU(5) grand unified theories, Phys. Rev. Lett. 43 (1979) 893.

[17] J.R. Ellis, M.K. Gaillard and D.V. Nanopoulos, On the effective lagrangian for baryon decay, Phys. Lett. B 88 (1979) 320.

[18] Y. Achiman and M. Richter, Gauge mediated proton decay in a renormalizable SUSY SO(10) with realistic mass matrices, Phys. Lett. B 523 (2001) 304 hep-ph/0107055.

[19] J.R. Ellis, D.V. Nanopoulos and J. Walker, Flipping SU(5) out of trouble, Phys. Lett. B 550 (2002) 99 hep-ph/0205336.

[20] P.F. Pérez, Fermion mixings vs $D=6$ proton decay, Phys. Lett. B 595 (2004) 476 hep-ph/0403286. 
[21] G. Altarelli and F. Feruglio, SU(5) grand unification in extra dimensions and proton decay, Phys. Lett. B 511 (2001) 257 hep-ph/0102301.

[22] L.J. Hall and Y. Nomura, Gauge unification in higher dimensions, Phys. Rev. D 64 (2001) 055003 hep-ph/0103125.

[23] Y. Nomura, Strongly coupled grand unification in higher dimensions, Phys. Rev. D 65 (2002) 085036 hep-ph/0108170.

[24] A. Hebecker and J. March-Russell, Proton decay signatures of orbifold guts, Phys. Lett. B 539 (2002) 119 hep-ph/0204037.

[25] T. Asaka, W. Buchmüller and L. Covi, Quarks and leptons between branes and bulk, Phys. Lett. B 563 (2003) 209 hep-ph/0304142.

[26] T. Kobayashi, S. Raby and R.-J. Zhang, Constructing 5D orbifold grand unified theories from heterotic strings, hep-ph/0403065.

[27] S. Förste, H.P. Nilles, P.K.S. Vaudrevange and A. Wingerter, Heterotic brane world, hep-th/0406208.

[28] T. Asaka, W. Buchmüller and L. Covi, Gauge unification in six dimensions, Phys. Lett. B 523 (2001) 199 hep-ph/0108021.

[29] L.J. Hall, Y. Nomura, T. Okui and D.R. Smith, SO(10) unified theories in six dimensions, Phys. Rev. D 65 (2002) 035008 hep-ph/0108071.

[30] T. Asaka, W. Buchmüller and L. Covi, Bulk and brane anomalies in six dimensions, Nucl. Phys. B 648 (2003) 231 hep-ph/0209144.

[31] For a recent review, see C.A. Scrucca and M. Serone, Anomalies in field theories with extra dimensions, Int. J. Mod. Phys. A 19 (2004) 2579 hep-th/0403163.

[32] R. Fleischer, CP-violation in the $B$ system and relations to $k \rightarrow \pi \nu \bar{\nu}$ decays, Phys. Rept. 370 (2002) 537 hep-ph/0207108.

[33] H. Fritzsch, Z. Xing, Mass and flavor mixing schemes of quarks and leptons, Prog. Part. Nucl. Phys. 45 (2000) 1 hep-ph/9912358.

[34] A. Hebecker, 5D super Yang-Mills theory in 4D superspace, superfield brane operators and applications to orbifold guts, Nucl. Phys. B 632 (2002) 101 hep-ph/0112230.

[35] T. Yanagida and J. Sato, Large lepton mixing in seesaw models: coset-space family unification, Nucl. Phys. 77 (Proc. Suppl.) (1999) 293 hep-ph/9809307.

[36] G. Altarelli and F. Feruglio, Models of neutrino masses and mixings, New J. Phys. 6 (2004) 106 hep-ph/0405048.

[37] C.D. Froggatt and H.B. Nielsen, Hierarchy of quark masses, cabibbo angles and CP-violation, Nucl. Phys. B 147 (1979) 277.

[38] W. Buchmüller and T. Yanagida, Quark lepton mass hierarchies and the baryon asymmetry, Phys. Lett. B 445 (1999) 399 hep-ph/9810308.

[39] M. Claudson, M.B. Wise and L.J. Hall, Chiral lagrangian for deep mine physics, Nucl. Phys. B 195 (1982) 297.

[40] S. Chadha and M. Daniel, Chiral lagrangian calculation of nucleon decay modes induced by $D=5$ supersymmetric operators, Nucl. Phys. B 229 (1983) 105. 
[41] N. Cabibbo, E.C. Swallow and R. Winston, Semileptonic hyperon decays, Ann. Rev. Nucl. Part. Sci. 53 (2003) 39 hep-ph/0307298.

[42] CP-PACS collaboration, N. Tsutsui et al., Lattice QCD calculation of the proton decay matrix element in the continuum limit, hep-lat/0402026.

[43] JLQCD collaboration, S. Aoki et al., Nucleon decay matrix elements from lattice QCD, Phys. Rev. D 62 (2000) 014506 hep-lat/9911026. 